\title{
Thyrotoxicosis, myasthenia gravis and periodic paralysis in a Chinese man
}

\author{
K.O. Lee, R. Guan, B. Ee and J.S. Cheah \\ University Department of Medicine, Singapore General Hospital, Singapore 0316.
}

\begin{abstract}
Summary: The association of myasthenia gravis and periodic paralysis in thyrotoxicosis has not been reported before. We describe a Chinese man with thyrotoxicosis and myasthenia gravis, who subsequently developed periodic paralysis. The possible aetiological links are discussed.
\end{abstract}

\section{Introduction}

Myasthenia gravis and periodic paralysis are both unusual complications of thyrotoxicosis. Although in Singapore periodic paralysis is relatively more common and occurs in $6 \%$ of all thyrotoxic patients, myasthenia gravis is less common. Both conditions are disorders affecting skeletal muscle. It is perhaps surprising therefore that the association of both myasthenia and periodic paralysis with thyrotoxicosis has not been described before. We describe one such patient here.

\section{Case report}

A male Chinese student was diagnosed at the age of $19 \mathrm{y}$ as having thyrotoxicosis. He had been attending regular follow-up for aortic regurgitation which was diagnosed when he was $10 \mathrm{y}$ old. His thyroid function was investigated when he complained of heat intolerance and had persistent tachycardia. Serum thyroxine (T4) was $13.1 \mu \mathrm{g} / \mathrm{dl}$ (normal range 3-7 $\mu \mathrm{g}$ / dl) and protein bound iodine (PBI) was $12 \mu \mathrm{g} / \mathrm{dl}$ (normal range 4-8 $\mu \mathrm{g} / \mathrm{dl}$ ). He was started on carbimazole and became euthyroid. His cardiac condition was stable.

Fourteen months later, he complained of a drooping right eyelid. An edrophonium (Tensilon) test was positive and a diagnosis of myasthenia gravis was made. Electromyography was done and reported as showing myasthenia changes in the extraocular muscles and the hypothenar muscles. He was started on

K.O. LEE, B.Sc., M.R.C.P. (UK); R. GUAN, M.R.C.P. (UK); B. Ee, M.R.C.P. (UK); J.S. Cheah, M.D., F.R.A.C.P. Correspondence: K.O. Lee, University Department of Medicine (II), Singapore General Hospital, Singapore 0316. Accepted: 2 November 1983 pyridostigmine and there was significant improvement. His carbimazole was stopped.

One year later, at the age of 21 years, he had aortic valve replacement and a bicuspid aortic valve was found. A thymectomy was done at the same time in view of his myasthenia. Histological study of the thymus showed clear demarcation of cortical and medullary zones with a number of prominent germinal centres. These features were reported as consistent with thymic follicular hyperplasia of myasthenia gravis. Post operative recovery was uneventful and one month after surgery, he was taken off digoxin and diuretics. He remained well and was followed up at regular intervals of 6 months. He was on warfarin $4 \mathrm{mg}$ daily and pyridostigmine $180 \mathrm{mg}$ daily in divided doses.

Two and a half years after his surgery, at the age of 23 , he was admitted with severe lower limb weakness and mild upper limb weakness. He had noticed mild weakness 2 days before admission in all 4 limbs and this deteriorated suddenly over the preceding 12 hours. On questioning, he also gave a 3 week history of increased sweating, palpitations and some weight loss.

On clinical examination, he was thyrotoxic. He had a fine tremor in his outstretched hands. Pulse was 96 beats/min. The mild ptosis of his right eyelid was unchanged. Power was decreased equally in both lower limbs (MRC Grade 3/5) especially in the flexor muscles. In both upper limbs, there was decreased power (MRC Grade 3/5) in the proximal muscles. Fatiguability was not present. A diagnosis of thyrotoxic periodic paralysis was made.

Serum T4 was $29 \mu \mathrm{g} / \mathrm{dl}$ (normal 4.6-12) $\mathrm{T}_{3}$ resin uptake $156 \%$ (normal $77-129 \%$ ) and FTI 45.8 (normal 4.6-11.6). Serum potassium was low at $1.6 \mathrm{mmol} / 1$. Other electrolytes and biochemical investigations (including muscle enzymes) were normal. 
Thyroid anti-microsomal antibodies were positive but anti-thyroglobulin antibodies were negative. His HLA profile was A2 A9/Bw $22 \mathrm{Bw} 46$.

Oral potassium supplements and carbimazole were started. His muscle weakness improved. No change was made in his pyridostigmine and he continued on $180 \mathrm{mg}$ daily. Slight drooping of his right eyelid persisted. He became euthyroid with treatment and continued on oral potassium supplements.

At follow-up nine months later, he was well. There was no weakness in his limbs. He was clinically euthyroid. Drooping of his right eyelid was unchanged.

\section{Discussion}

As far as we know, this is the first reported association of myasthenia gravis and periodic paralysis in a thyrotoxic patient. Fourteen months after the onset of thyrotoxicosis he developed myasthenia gravis. The diagnosis of this was confirmed by the edrophonium test and by electromyography. Drachman (1962) and Gaelen and Levitan (1968) have suggested that myasthenia is worsened by any deviation from the euthyroid state. The myasthenia in this patient remained very mild and easily controlled despite his relapse of thyrotoxicosis. The thymectomy done so early on in his disease might be the reason for the mildness of his myasthenia.

Sudden onset of weakness in a patient with myasthenia gravis could be due to a myasthenic or a cholinergic crisis. However the features of thyrotoxic periodic paralysis (TPP) are sufficiently different for the diagnosis to be made with certainty. In Singapore, the typical patient with TPP is a male Chinese patient aged 20-29. Thyrotoxicosis precedes or is diagnosed at the same time as the onset of paralysis. There is marked weakness of the lower limbs but fatiguability and eyelid involvement do not occur. Hypokalaemia is present in patients with TPP (Cheah, 1978; McFad-

\section{References}

CHEAH, J.S. (1978). Thyrotoxic periodic paralysis in Singapore. Proceedings of the 6th Asia and Oceanic Congress in Endocrinology, Singapore 1978, 1, 282.

DRACHMAN, D.B. (1962). Myasthenia gravis and the thyroid gland, New England Journal of Medicine, 266, 330.

GAELEN, L.H. \& LEVITAN, S. (1968). Myasthenia gravis and thyroid function. Archives of Neurology, 18, 107.

GARLEPP, M.J., CHRISTIANSEN, F.T., DAWKINS, R.L., CHIEWSLIP, P. \& TAKATA, H. (1982). Myasthenia gravis. In Proceedings of D-Pen HLA 82 Workshop, Perth, Australia p. 259-263, Excerpta Medica. Amsterdam.

McFADZEAN, A.J.S. \& YEUNG, R. (1967). Periodic paralysis complicating thyrotoxicosis in Chinese. British Medical zean and Yeung, 1967). All the typical features of TPP were found in this patient.

We believe his cardiac condition was not related to his other diagnoses.

Thyrotoxic periodic paralysis is relatively common and occurs in $6 \%$ of all thyrotoxic patients in Singapore. The mechanism remains unclear but the basic defect is thought to lie in the skeletal muscle surface membrane. This defect is persistent throughout life and paralysis can be induced in TPP patients who have been rendered euthyroid and are then deliberately made thyrotoxic by administration of thyroxine (Okihiro \& Nordyke, 1966).

Myasthenia gravis is known to be due to autoimmune damage to acetylcholine receptors at the neuromuscular junction of skeletal muscle, in which auto-antibody is implicated. It is widely accepted that there is an association with thyroid disease. Simpson (1968) has suggested that this association is probably genetic and immunological. There are no published figures of the association of thyrotoxicosis and myasthenia in Singapore but the clinical impression is that about $5-10 \%$ of myasthenia patients have thyrotoxicosis.

HLA studies on patients with thyrotoxicosis and periodic paralysis have suggested a genetic link (Yeo et al., 1978). There is a significant increase in HLA Bw 46 in Chinese with Graves' disease. HLA A2 Bw 22 are significantly increased in patients with thyrotoxis periodic paralysis. Our patient has all these 3 HL antigens. In HLA studies on patients with myasthenia gravis, HLA B8 has been consistently increased significantly in all racial groups but was absent in our patient. In Thai patients, on the other hand, HLA Bw 46 is also associated with early onset generalized myasthenia gravis (Garlepp et al., 1982). Our patient has an HLA profile of HLA A2 A9/Bw $22 \mathrm{Bw} 46$ and this would further support the concept of a genetic cause for the association of both periodic paralysis and myasthenia gravis with thyrotoxicosis.

Journal, 1, 451.

OKIHIRO, M.M. \& NORDYKE, R.A. (1966). Hypokalaemic periodic paralysis, Experimental precipitation with sodium liothyronine. American Journal of Medicine, 198, 949.

SIMPSON, J.A. (1968). The correlations between myasthenia gravis and disorders of the thyroid gland. In Research in Muscular Dystrophy. Proceedings of the 4th Symposium, p. 31-41, Pitman, London.

YEO, P.P.B., CHAN, S.H., LIU, K.F., WEE, G.B., LIM, P. \& CHEAH, J.S. (1978). HLA and periodic paralysis, British Medical Journal, 2, 930. . 